\title{
Long non-coding RNA PTENP1 inhibits proliferation and migration of breast cancer cells via AKT and MAPK signaling pathways
}

\author{
SHENG CHEN $^{1 *}$, YE WANG $^{2 *}$, JIAN-HUA ZHANG $^{1}$, QI-JUN XIA ${ }^{1}$, QIANG SUN $^{1}$, \\ ZHEN-KAI LI ${ }^{1}$, JIAN-GUO ZHANG ${ }^{1}$, MAO-SHENG TANG ${ }^{1}$ and MAO-SHENG DONG ${ }^{1}$ \\ ${ }^{1}$ Department of General Surgery, The General Hospital of the PLA Rocket Force, Beijing 100088; \\ ${ }^{2}$ Department of Pathology, China-Japan Friendship Hospital, Beijing 100029, P.R. China
}

Received May 15, 2017; Accepted August 18, 2017

DOI: $10.3892 / \mathrm{ol} .2017 .6823$

\begin{abstract}
We aimed to investigate the influence of long non-coding RNA (lncRNA) PTEN pseudogene-1 (PTENP1) on the proliferation, migration and cycle of breast cancer cells and its mechanism. Lentiviral vectors expressing PTENP1 were synthesized and breast cancer cells MCF7 were transfected with LV003-GFP-PTENP1 and LV003-GFP, respectively. The proliferation capacities of breast cancer cells were detected using CCK-8 assay, and the migration capacities of breast cancer cells were detected using scratch assay; flow cytometry was used to detect the cell cycles and Western blot was used to detect the expression levels of cyclin A2, CDK2, p-p44/42 MAPK, t-p44/42 MAPK, p-p38 MAPK, t-p38 MAPK, p-AKT, t-AKT in AKT and MAPK pathways. The absorbance values (A450) of cells in experimental group at 48 and $72 \mathrm{~h}$ were $1.4 \pm 0.3$ and $2.3 \pm 0.47$, respectively, which were significantly lower than those in control group $(3.2 \pm 0.39$, $3.4 \pm 0.58)(\mathrm{P}<0.05)$. The number of cell colonies in experimental group was $(48 \pm 13)$, which was significantly lower than that in control group $(159 \pm 16)(\mathrm{P}<0.01)$. The cell migration rate in experimental group was $22.8 \pm 3.3 \%$, which was significantly lower than that in control group $61.8 \pm 5.2 \%(\mathrm{P}<0.01)$. Western blot detection showed that the expression levels of cyclin A2, CDK2, p-AKT, p-p44/42 MAPK and p-p38 MAPK in experimental group were significantly decreased compared with those in control group. LncRNA PTENP1 can inhibit the proliferation and migration of breast cancer cells via the AKT and MAPK signaling pathways.
\end{abstract}

Correspondence to: Dr Mao-Sheng Dong, Department of General Surgery, The General Hospital of the PLA Rocket Force, 16 Xinjie Kouwai Street, Xicheng, Beijing 100088, P.R. China E-mail: dongmaosheng886@163.com

*Contributed equally

Key words: lncRNA, PTENP1, breast cancer, cell cycle, migration

\section{Introduction}

Breast cancer is a major disease threatening women's health. According to statistics, breast cancer accounts for one-third in all new-onset tumors in female (1). The occurrence and development of breast cancer are multi-gene and multi-step chain processes, which is promoted by gene abnormality; besides, abnormal epigenetic regulation also plays an important role in the occurrence process of breast cancer (2). In recent years, studies have found that long non-coding RNA (lncRNA) plays an important role in the occurrence and development of breast cancer through the epigenetic regulation mechanism (3).

PTEN (phosphatase and tensin homolog deleted on chromosome ten) has been reported to inhibit progression and development of various cancers, and it was established as a tumor suppressor (4). For instance, PTEN can participate in suppressing the proliferation of cancer cells via negatively regulating the PI3K/AKT pathway $(5,6)$, which suggests that PTEN is important in malignant transformation of cancer cells. However, little is known about the detailed mechanism how PTEN is changed during carcinogenesis. In recent years, non-coding RNA (ncRNA) has drawn increasing attention in the field of cancer. As reported, PTENp1 (PTEN pseudogene 1) could regulate the expression of the ancestral gene PTEN, thus exerted an effect on the process of carcinogenesis (7). However, the function and mechanism of PTEN pseudogene 1 (PTENP1) in breast cancer cells have not been completely clarified. Breast cancer cell lines expressing lncRNA PTENP1 stably were established in this study, so as to investigate the role of PTENP1 in the proliferation and migration of breast cancer cells and its mechanism.

\section{Materials and methods}

Materials. Breast cancer cell line MCF7 and cell line 293T used for lentivirus packaging were purchased from Institute of Biochemistry and Cell Biology, Shanghai Institutes for Biological Sciences, Chinese Academy of Sciences. TRIzol reagent was purchased from Invitrogen (Carlsbad, CA, USA), trypsin and fetal bovine serum DMEM were purchased from 
Gibco (Grand Island, NY, USA), dimethyl sulfoxide was from Sigma (St. Louis, MO, USA), CCK-8 kit was from Dojindo (Kumamoto, Japan), RNA extraction kit and TRIzol were obtained from Takara (Otsu, Japan), and the rabbit anti-human p-p44/42MAPK, p-p38 MAPK, p-AKT, cyclin A2, CDK2 and mouse anti-human GAPDH monoclonal antibodies were purchased from Cell Signaling Technology (Beverly, MA, USA).

Cell culture. MCF7 cells were cultured in DMEM medium containing $10 \%$ fetal bovine serum and the medium was placed in an incubator containing $5 \% \mathrm{CO}_{2}$ at $37^{\circ} \mathrm{C}$. Cells were digested by $0.25 \%$ trypsin, followed by passage; and cells in logarithmic phase were used for the experiment.

Construction of IncRNA PTENPI lentiviral expression vector. According to DNA sequence of PTENP1 in National Center for Biotechnology Information (NCBI), the target gene PTENP1-3'UTR was amplified with DNA in human skin tissue as the template. Gene primer sequences were as follows: sense strand: 5'-AGTCACCTGTTAAGAAAATGAGAA GACAAA-3', antisense strand: 5'-CTGTCCCTTATCAGA TACATGACTTTCAA-3'. The recombinants expressing PTENP1 were constructed by LV003-GFP vector, followed by sequencing and identification.

Lentivirus packaging. Four kinds of plasmids (pspax2, pMD2G, pLVX-IRES-ZsGreen1 and pLV003-PTENP1) in lentiviral packaging system were prepared; ZsGreen1 expression cassette on the plasmid could express GFP; 293T cells were transfected with the plasmids containing the target gene and the four kinds of plasmids in lentiviral packaging system for virus packaging, and the virus supernatant was collected; the supernatant was centrifuged in a $40 \mathrm{ml}$ ultraspeed centrifuge tube at $80,000 \mathrm{xg}$ for $2 \mathrm{~h}$ at $4^{\circ} \mathrm{C}$; the in a $40 \mathrm{ml}$ ultracentrifuge tube. The virus precipitation was resuspended using iced PBS, dissolved at $4^{\circ} \mathrm{C}$ overnight and stored at $-80^{\circ} \mathrm{C}$.

Construction of breast cancer cell line MCF7 expressing lncRNA PTENPI stably. When the cell fusion of MCF7 reached 60-70\%, GFP-PTENP1 and LV003-GFP were added on the cell surface at the multiplicity of infection $(\mathrm{MOI})=10$, respectively. After $8 \mathrm{~h}$, the normal DMEM medium containing $10 \%$ fetal calf serum was used. The experimental group (MCF7 cells transfected with LV003-GFP-PTENP1) and control group (MCF7 cells transfected with LV003-GFP) were set up. Puromycin $(1 \mu \mathrm{l} / \mathrm{ml})$ was added for screening after green fluorescence was observed under the fluorescence microscope, fluorescence photos were taken after 3 days, and the expression of PTENP1 was detected by real-time fluorescent quantitative PCR.

Detection of proliferation capacity of breast cancer cells via $C C K$-8. Cells taken from the two groups were inoculated onto the 96 -well plates $\left(2 \times 10^{3} /\right.$ well) with 5 repetition wells in each group. CCK-8 solution was added with $10 \mu \mathrm{l}$ /well at 12,24 , 48 and $72 \mathrm{~h}$, respectively, and the cells were incubated in an incubator for $2 \mathrm{~h}$. A450 was measured with the microplate reader, and the growth curve was drawn according to A450s.

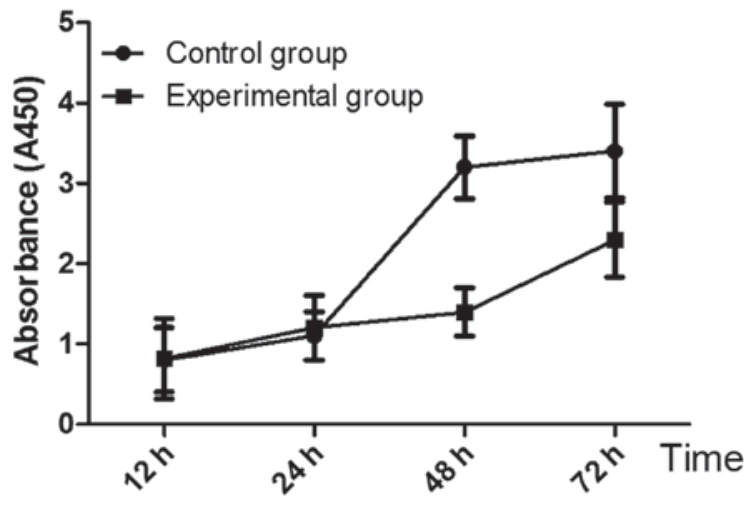

Figure 1. The growth curve of breast cancer MCF7 cells.

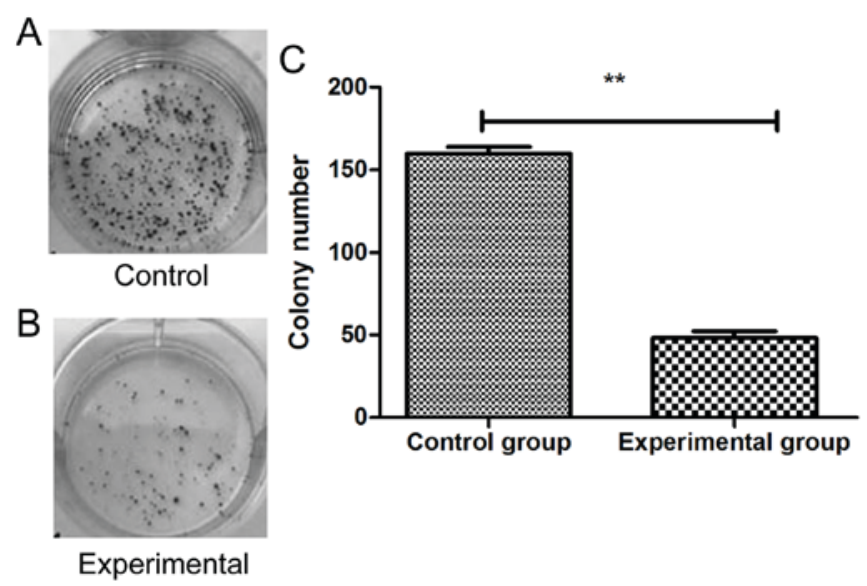

Figure 2. Colony-forming assay of breast cancer MCF7 cells. (A) Control group; (B) experimental group; (C) compared with control group, colony number was significantly reduced in the experimental group $\left.{ }^{* *} \mathrm{P}<0.01\right)$.

Detection of clone formation capacity of breast cancer cells via plate clone assay. Cells taken from experimental group and control group were digested to prepare the single-cell suspension, and the cell density was adjusted to $2 \times 10^{3} / \mathrm{ml}$, and $100 \mu \mathrm{l}$ cell suspension was taken and inoculated onto the 6-well plate, and then $2 \mathrm{ml}$ medium was added. The cell suspension was cultured in an incubator for 1-2 weeks and was seeded when the clone formation was visible, followed by Giemsa staining; and the number of clones was counted.

Detection of migration rate of breast cancer cells via scratch assay. The above-mentioned cells were inoculated onto the 6-well plate, and when the cell fusion reached $100 \%$, a straight line was drawn in the middle of each well with 1-ml spearhead, and the photos of cells were taken after $24 \mathrm{~h}$; the area change before and after the scratch was calculated. Cell migration rate $(\%)=($ area in scratch - area after $24 \mathrm{~h} /$ area in scratch) $\mathrm{x} 100 \%$.

Detection of protein expression via western blot method. Cells in logarithmic phase were taken from experimental group and control group; the total protein of each group was extracted and quantified according to the procedures in protein extraction kit. The protein was transferred to polyvinylidene chloride membrane by wet transfer method after 


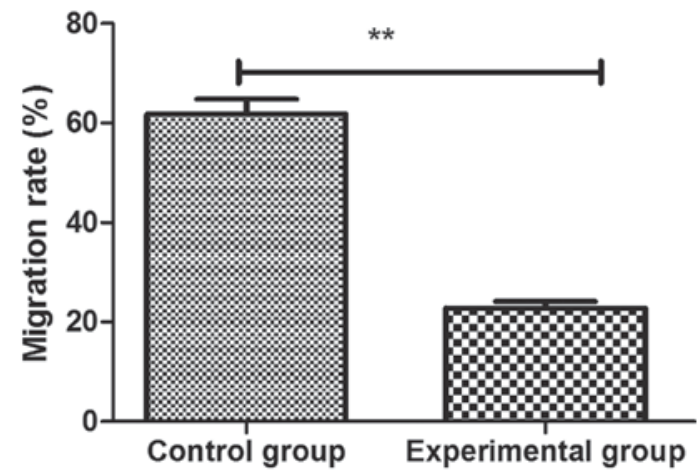

Figure 3. Influence of lncRNA PTENP1 on migration capacity of breast cancer MCF7 cells between control group and experimental group $\left({ }^{* * *} \mathrm{P}<0.01\right)$

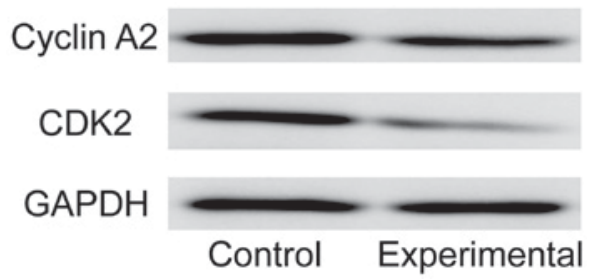

Figure 4. Influence of 1ncRNA PTENP1 on cyclin of breast cancer MCF7 cells between control group and experimental group.

acrylamide gel electrophoresis. The membrane was sealed by skimmed milk for $1 \mathrm{~h}$. And then primary rabbit monoclonal p-AKT antibody (dilution, 1:500; cat. no. ab81283); rabbit polyclonal t-AKT antibody (dilution, 1:500; cat. no. ab38449); rabbit monoclonal p-P44/42MAPK antibody (dilution, 1:500; cat. no. ab53277); rabbit monoclonal t-P44/42 MAPK antibody (dilution, 1:500; cat. no. ab50011); rabbit polyclonal p-p38 MAPK antibody (dilution, 1:500; cat. no. ab47363); rabbit polyclonal t-p38 MAPK antibody (dilution, 1:500; cat. no. ab197348); rabbit monoclonal cyclinA2 antibody (dilution, 1:500; cat. no. ab32386); rabbit monoclonal CDK2 antibody (dilution, 1:500; cat. no. ab32147); rabbit polyclonal GAPDH antibody (dilution, 1:500; cat. no. ab37168) were added. All the antibodies were all purchased from Abcam (Cambridge, MA, USA). Then the protein was incubated overnight at $4^{\circ} \mathrm{C}$. After the protein was washed with TBST three times, secondary goat anti-rabbit (HRP) IgG antibody (dilution, 1:2,000; cat. no. ab6721) was added for incubation for $1 \mathrm{~h}$, followed by development via enhanced chemiluminescence (ECL).

Statistical analysis. SPSS 17.0 software (SPSS, Inc., Chicago, IL, USA) was used for data analysis. The t-test was used for the comparison of protein expression levels and absorbance values between the two groups. $\mathrm{P}<0.05$ was considered to indicate a statistically significant difference.

\section{Results}

Influence of IncRNA PTENP1 on proliferation capability of breast cancer MCF7 cells. The growth curve of breast cancer MCF7 cells is shown in Fig. 1. A450 of cells in experimental group at $48 \mathrm{~h}$ and $72 \mathrm{~h}$ was $1.4 \pm 0.3$ and $2.3 \pm 0.47$, respectively, which were significantly lower than those in control group

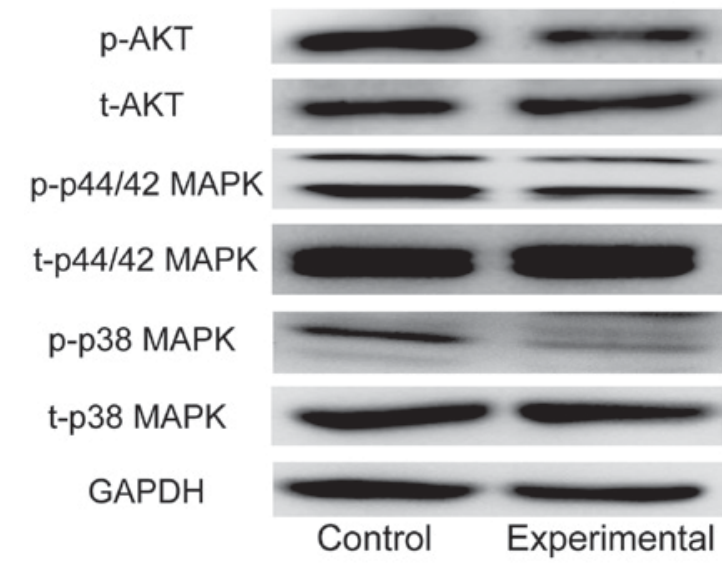

Figure 5. Influence of lncRNA PTENP1 on AKT and MAPK signaling pathways of breast cancer MCF7 cells between control group and experimental group.

$(3.2 \pm 0.39,3.4 \pm 0.58)(\mathrm{P}<0.05)$. Colony-forming assay is shown in Fig. 2, and the results showed that the number of cell clones in experimental group was $48 \pm 13$, which was significantly lower than that in control group $(159 \pm 16)(\mathrm{P}<0.01)$.

Influence of IncRNA PTENP1 on migration capacity of breast cancer MCF7 cells. Scratch assay showed that the cell migration rate in experimental group was $22.8 \pm 3.3 \%$, which was significantly lower than that in control group $61.8 \pm 5.2 \%$ $($ p $<0.01)$ (Fig. 3).

Influence of IncRNA PTENPI on cyclin of breast cancer MCF 7 cells. Cyclin A2 and CDK2 are the important proteins that promote cells to transform from $S$ phase to $\mathrm{G} 2$ phase. Western blot detection showed that the expression levels of cyclin A2 and CDK2 in experimental group were significantly lower than those in control group (Fig. 4).

Influence of IncRNA PTENP1 on AKT and MAPK signaling pathways. Western blot detection showed that the expression of p-AKT protein, an important factor in AKT signaling pathway, in experimental group was significantly decreased compared with that in control group. In addition, the protein expression levels of p-p38 MAPK and p-p44/42 MAPK, important factors in MAPK signaling pathway, in experimental group were significantly decreased compared with those in control group (Fig. 5).

\section{Discussion}

lncRNA is a transcription RNA with the length of more than 200 nucleotides. IncRNA can not be translated into protein because of lack of a promoter. More and more studies have confirmed that lncRNA regulates the proliferation, apoptosis, differentiation and migration of tumor cells through its participation in regulating chromatin modification, transcriptional activation, intranuclear interference and silencing of $\mathrm{X}$ chromosome (8). Studies have shown that lncRNA also plays an important regulatory role in the occurrence and development of breast cancer. In ER $\alpha$-positive breast cancer, IncRNA H19 can improve the chemotherapy resistance of breast cancer 
by downregulating BIK, a pro-apoptotic gene (9). In breast cancer cells, long non-coding RNA UCA1 can enhance the chemotherapy resistance of tamoxifen, which may be realized through the regulation of miR-18a-HIF1 $\alpha$ feedback loop (10). In this study, breast cancer cell MCF7 expressing PTENP1 was established. CCK-8 proliferation assay and plate clone assay showed that PTENP1 could inhibit the proliferation and growth of breast cancer cells, scratch assay showed that PTENP1 could inhibit the migration of breast cancer cells.

Via directly inhibiting phosphoinositide 3-kinase (PI3K) signaling pathway and negatively regulating miR-21, PTEN could affect a series of cellular processes $(11,12)$. As showed in our study, PTENp1 inhibited cell proliferation and migration via AKT signaling pathway as well as cell cycle related proteins such as cyclin A2 and CDK2. This discovery revealed that PTENp1, a kind of pseudogene transcript that blocks miRNA activity in breast cancer, could post-transcriptionally regulate many potential biological roles.

MAPK is a serine/threonine protein kinase in cells, which plays an important role in breast cancer. Abnormal changes of MAPK signaling pathway play an important role in the growth, differentiation and other physiological activities of breast cancer. To investigate the role of PTENP1 in MAPK signaling pathway in breast cancer cells, the protein expression levels of p-p44/42 MAPK and p-p38 MAPK in MCF7 expressing PTENP1 were detected, and it was found that PTENP1 could downregulate the phosphorylation of Erk1/2 (P44/42 MAPK) and p38 MAPK protein, important factors in MAPK signaling pathway in breast cancer cells, indicating that PTENP1 can also regulate the proliferation and migration of breast cancer cells via regulating MAPK signaling pathway, in addition to inhibiting the activity of AKT signaling pathway in breast cancer cells.

In recent years, some scholars have proposed the competitive endogenous RNA (ceRNA) hypothesis; in other words, there are common microRNA binding sites in IncRNA and mRNA in corresponding functional network, which weakens the inhibiting effect of microRNA on homologous mRNA via microRNA adsorption (13-15). Studies show that lncRNA PTENP1 can also regulate the expression of PTEN through the method in ceRNA hypothesis, thus affecting the progression of tumor cells (16-18). Whether the mechanism of ceRNA is involved in breast cancer cells needs further study.

In conclusion, lncRNA PTENP1 can inhibit the proliferation and migration of breast cancer cells, which may be realized through downregulating cyclin $\mathrm{A} 2$ and CDK2, the important proteins in cell cycle, and AKT and MAPK signaling pathways. IncRNA PTENP1 plays an important role in the occurrence and development of breast cancer, and the in-depth study on it can reveal not only the related mechanism of PTEN, its cancer suppressor gene, but also the role and mechanism of lncRNA in breast cancer. In addition, the in-depth study on IncRNA can provide new ideas for the prevention and treatment of breast cancer.

\section{References}

1. Shi Y, Yang F, Sun Z, Zhang W, Gu J and Guan X: Differential microRNA expression is associated with androgen receptor expression in breast cancer. Mol Med Rep 15: 29-36, 2017.

2. Marzese DM, Gago FE, Vargas-Roig LM and Roqué M: Methylation profile of human breast cancer: A possible biomarker for the detection of circulating tumor cells. J Clin Oncol 27 (Suppl 15): 11112, 2009.

3. Yu X and Li Z: Long non-coding RNA HOTAIR: A novel oncogene (Review). Mol Med Rep 12: 5611-5618, 2015.

4. Perez-Tenorio G, Alkhori L, Olsson B, Waltersson MA, Nordenskjöld B, Rutqvist LE, Skoog L and Stål O: PIK3CA mutations and PTEN loss correlate with similar prognostic factors and are not mutually exclusive in breast cancer. Clin Cancer Res 13: 3577-3584, 2007.

5. Stambolic V, Suzuki A, de la Pompa JL, Brothers GM, Mirtsos C, Sasaki T, Ruland J, Penninger JM, Siderovski DP and Mak TW: Negative regulation of PKB/Akt-dependent cell survival by the tumor suppressor PTEN. Cell 95: 29-39, 1998.

6. Meng F, Henson R, Wehbe-Janek H, Ghoshal K, Jacob ST and Patel T: MicroRNA-21 regulates expression of the PTEN tumor suppressor gene in human hepatocellular cancer. Gastroenterology 133: 647-658, 2007.

7. Karreth FA, Tay Y, Perna D, Ala U, Tan SM, Rust AG, DeNicola G, Webster KA, Weiss D, Perez-Mancera PA, et al: In vivo identification of tumor- suppressive PTEN ceRNAs in an oncogenic BRAF-induced mouse model of melanoma. Cell 147: 382-395, 2011.

8. Wilusz JE, Sunwoo H and Spector DL: Long noncoding RNAs: Functional surprises from the RNA world. Genes Dev 23: 1494-1504, 2009.

9. Si X, Zang R, Zhang E, Liu Y, Shi X, Zhang E, Shao L, Li A, Yang N, Han X, et al: LncRNA H19 confers chemoresistance in ER $\alpha$-positive breast cancer through epigenetic silencing of the pro-apoptotic gene BIK. Oncotarget 7: 81452-81462, 2016.

10. Li X, Wu Y, Liu A and Tang X: Long non-coding RNA UCA1 enhances tamoxifen resistance in breast cancer cells through a miR-18a-HIF1 $\alpha$ feedback regulatory loop. Tumour Biol 37: 14733-14743, 2016

11. Li J, Simpson L, Takahashi M, Miliaresis C, Myers MP, Tonks N and Parsons R: The PTEN/MMAC1 tumor suppressor induces cell death that is rescued by the AKT/protein kinase B oncogene. Cancer Res 58: 5667-5672, 1998.

12. Choi HJ, Chung TW, Kang SK, Lee YC, Ko JH, Kim JG and Kim CH: Ganglioside GM3 modulates tumor suppressor PTENmediated cell cycle progression - transcriptional induction of p21(WAF1) and p27(kip1) by inhibition of PI-3K/AKT pathway. Glycobiology 16: 573-583, 2006.

13. Yu G, Yao W, Gumireddy K, Li A, Wang J, Xiao W, Chen K, Xiao H, Li H, Tang K, et al: Pseudogene PTENP1 functions as a competing endogenous RNA to suppress clear-cell renal cell carcinoma progression. Mol Cancer Ther 13: 3086-3097, 2014.

14. Alimonti A, Carracedo A, Clohessy JG, Trotman LC, Nardella C, Egia A, Salmena L, Sampieri K, Haveman WJ, Brogi E, et al: Subtle variations in Pten dose determine cancer susceptibility. Nat Genet 42: 454-458, 2010.

15. Salmena L, Poliseno L, Tay Y, Kats L and Pandolfi PP: A ceRNA hypothesis: The Rosetta Stone of a hidden RNA language? Cell 146: 353-358, 2011.

16. Poliseno L, Salmena L, Zhang J, Carver B, Haveman WJ and Pandolfi PP: A coding-independent function of gene and pseudogene mRNAs regulates tumour biology. Nature 465: 1033-1038, 2010.

17. Johnsson P, Ackley A, Vidarsdottir L, Lui WO, Corcoran M, Grandér D and Morris KV: A pseudogene long-noncoding-RNA network regulates PTEN transcription and translation in human cells. Nat Struct Mol Biol 20: 440-446, 2013.

18. Chen CL, Tseng YW, Wu JC, Chen GY, Lin KC, Hwang SM and $\mathrm{Hu}$ YC: Suppression of hepatocellular carcinoma by baculovirusmediated expression of long non-coding RNA PTENP1 and MicroRNA regulation. Biomaterials 44: 71-81, 2015. 